\title{
Making the case for EGFR TKI sequencing in EGFR mutation-positive NSCLC: a GioTag study US patient analysis
}

\author{
Bruce Feinberg*,1(D), Balazs Halmos² (D), Rasim Gucalp² (iD, Wenbo Tang ${ }^{3}$, Barbara \\ Moehring $^{3}$ \& Maximillian J Hochmair ${ }^{4}$ (iD) \\ ${ }^{1}$ Cardinal Health, Dublin, $\mathrm{OH} 43017$, USA \\ ${ }^{2}$ Department of Oncology, Montefiore/Albert Einstein Cancer Center, Bronx, NY 10467, USA \\ ${ }^{3}$ Boehringer Ingelheim Pharmaceuticals Inc., Ridgefield, CT 06877, USA \\ ${ }^{4}$ Department of Respiratory \& Critical Care Medicine, Karl Landsteiner Institute of Lung Research \& Pulmonary Oncology, Vienna, \\ Austria \\ *Author for correspondence: Tel.: +1 404210 8788; Fax: +1 614553 9932; bruce.feinberg@cardinalhealth.com
}

Aim: To assess time-to-treatment failure (TTF) in US patients with epidermal growth factor receptor (EGFR) mutation-positive non-small-cell lung cancer (NSCLC) who received sequential afatinibosimertinib treatment in the global, observational GioTag study. Patients \& methods: Patients had EGFR T790M mutation-positive disease after first-line afatinib and subsequently received osimertinib. The primary outcome was TTF. Results: In 129 patients at US centers, median TTF was 28.4 months (90\% Cl: 27.0-34.1). Median overall survival was 47.6 months (90\% Cl: $35.5-51.5$ ). Conclusion: Sequential afatinib-osimertinib in this US-treated population was associated with long median TTF and represents an effective, evidence-based treatment option for US patients with EGFR mutation-positive NSCLC not presenting with active brain metastases or de novo T790M. Clinical Trial Registration: NCT03370770 (ClinicalTrials.gov)

First draft submitted: 3 March 2020; Accepted for publication: 7 July 2020; Published online: 6 August 2020

Keywords: afatinib • NSCLC • osimertinib • sequential treatment

Treatment with an EGFR tyrosine kinase inhibitor (TKI) is the evidence-based standard of care for patients with EGFR mutation-positive non-small-cell lung cancer (NSCLC), based on reports of prolonged progression-free survival (PFS) and improved tolerability with EGFR TKIs relative to chemotherapy [1-6]. In the US, three generations of EGFR TKI are available: the reversible first-generation TKIs erlotinib [7] and gefitinib [8], the irreversible secondgeneration ErbB family blockers dacomitinib [9] and afatinib [10] and the irreversible third-generation, EGFR wild-type sparing TKI osimertinib [11]. Several large randomized controlled studies have demonstrated improved efficacy outcomes with the second- [12-14] and third-generation TKIs $[15,16]$ and also more favorable tolerability with osimertinib [16], compared with the first-generation agents in EGFR mutation-positive NSCLC. Indeed, the dramatic improvement in PFS reported with osimertinib compared with gefitinib and erlotinib in the Phase III FLAURA study [16], as well as its activity against the EGFR T790M resistance mutation [17] and its ability to cross the blood-brain barrier [18-20], has distinguished osimertinib from the first-generation TKIs. Consequently, osimertinib has been rapidly adopted as a preferred first-line treatment for EGFR mutation-positive NSCLC; however, there are no prospective data comparing outcomes with osimertinib and second-generation TKIs and very few patients present with de novo T790M (when direct sequencing techniques are used) [21-23] or CNS metastases [24].

With all of the EGFR TKIs, acquired resistance to first-line treatment is inevitable, necessitating second-line treatment options. The most common mechanism of resistance to first- and second-generation EGFR TKIs is the emergence of T790M (reported in $\sim 60$ of all patients and up to $\sim 75 \%$ of patients with EGFR Del19 mutations using techniques including liquid biopsy digital droplet [dd] PCR) [25-28]. As shown by the AURA studies, osimertinib is a suitable treatment option for patients progressing with an EGFR T790M after first-line EGFR

Future Medicine 
TKI therapy [17,25,29] and has been approved by regulatory authorities in this setting [11]. Conversely, resistance mechanisms to first-line osimertinib appear to be heterogeneous and include MET, EGFR and KRAS amplification, KRAS or PIK3CA mutations, EGFR C797S mutation and small-cell transformation [30-32]. Due to the genomic complexity of tumors with EGFR mutation-positive NSCLC following first-line osimertinib, no standard targeted second-line treatment has been identified $[30,31]$ and at present is likely to be chemotherapy based or an experimental therapy [33]. There is rationale, therefore, for investigating sequential EGFR TKI treatment in patients with EGFR mutation-positive NSCLC, reserving osimertinib for second-line use and thereby prolonging chemotherapy-free treatment.

Data on the cumulative benefit of sequential EGFR TKIs in patients with EGFR mutation-positive NSCLC are limited $[17,25,29,34]$. Assessing outcomes in a 'real-world' treatment setting, the global GioTag study investigated use of osimertinib in patients who develop EGFR T790M-positive disease after first-line afatinib [35]. In the primary analysis (database lock: May 2018), median time-to-treatmentfailure (TTF, defined as time from start of afatinib to discontinuation of osimertinib, or death) was 27.6 months (90\% CI: $25.9,31.3$ ) and was 30.3 months ( $90 \%$ CI: 27.6-44.5) in patients with EGFR Del19 mutation-positive NSCLC [35]. An interim analysis from a later data snapshot (April 2019) has also been reported [36]. These real-world data indicate that sequential afatinib-osimertinib is an attractive treatment option for patients with $\mathrm{T} 790 \mathrm{M}$-acquired resistance and effectively prolongs the overall chemotherapy-free treatment period. Further, these data complement previous findings from the randomized clinical trial setting that provided rationale for further investigation of sequential afatinib-osimertinib treatment [34]. In a pooled analysis of patients with EGFR Del19/L858R mutation-positive NSCLC from the Phase III LUX-Lung 3 (afatinib vs cisplatin plus pemetrexed) and 6 (afatinib vs cisplatin plus gemcitabine) and Phase IIb LUX-Lung 7 (afatinib vs gefitinib) studies, 37 patients received osimertinib as $\geq$ second-line treatment and the 3-year overall survival (OS) rate in these patients was $\sim 90 \%$.

To ensure maximal survival benefit for individual patients treated in real-world clinical practice, it is important to confirm treatment outcomes in different patient subgroups. In the primary GioTag analysis, TTF was particularly encouraging in patients with tumors harboring an EGFR Del19 activating mutation and also in Asian patients [35]. Here, we report a subgroup analysis of patients from the GioTag study who were treated in the US, aiming to provide further insight into the outcomes associated with sequential afatinib-osimertinib treatment in US clinical practice.

\section{Materials \& methods}

Study design \& patients

The GioTag study (ClinicalTrials.gov: NCT03370770) has previously been reported in detail [35]. This was a global observational study in which medical and electronic health records of consecutive patients treated in everyday clinical practice were reviewed. A maximum of 15 patients were enrolled per site. Patients had advanced/metastatic NSCLC harboring common EGFR mutations (Del19/L858R) and had acquired an EGFR T790M mutation after first-line afatinib; they then received second-line osimertinib. Patients with active baseline brain metastases were excluded. Patients had stopped first-line afatinib and started osimertinib treatment $\geq 10$ months prior to enrollment to avoid early censoring and to ensure data maturity. The study was conducted in compliance with the principles of the Declaration of Helsinki, in accordance with the International Conference on Harmonization, Harmonized Tripartite Guideline for Good Clinical Practice, Good Epidemiological Practice, Guidelines for Good Pharmacoepidemiology Practice and relevant sponsor Standard Operating Procedures. All patients provided written, informed consent where required.

\section{Outcomes \& statistics}

The primary outcome was time on treatment, reported here as TTF (time from the start of afatinib to the discontinuation of osimertinib or death). TTF and OS were estimated using Kaplan-Meier methodology.

Data for this US subgroup analysis were sourced from medical and electronic records of consecutive patients treated at US treatment centers between 28 December 2017 and 31 May 2018 (database lock: 31 May 2018). Data were supplied by Cardinal Health (OH, US), Montefiore/Albert Einstein Cancer Center and Cancer Alliance of Nebraska. 
Table 1. Baseline demographics, disease characteristics and treatment starting dose in patients from US centers.

\begin{tabular}{|c|c|c|}
\hline Characteristic & At start of afatinib therapy $(n=129)$ & At start of osimertinib therapy $(n=129)$ \\
\hline Median age, years (range) & $60(52-66)$ & \\
\hline $\begin{array}{l}\text { Age: } \\
-<65 \text { years } \\
-\geq 65 \text { years }\end{array}$ & $\begin{array}{l}91(70.5) \\
38(29.5)\end{array}$ & \\
\hline Male, n (\%) & $64(49.6)$ & \\
\hline $\begin{array}{l}\text { Race: } \\
\text { - White } \\
\text { - Black or African American } \\
\text { - Asian } \\
\text { - Other } \\
\dagger\end{array}$ & $\begin{array}{l}81(62.8) \\
18(14.0) \\
19(14.7) \\
11(8.5)\end{array}$ & \\
\hline $\begin{array}{l}\text { Mutation type: } \\
\text { - Del19 only } \\
\text { - L858R only } \\
\text { - Del19+L858R }\end{array}$ & $\begin{array}{l}91(70.5) \\
37(28.7) \\
1(0.8)\end{array}$ & \\
\hline $\begin{array}{l}\text { Disease stage: } \\
\text { - IIIB } \\
\text { - IV }\end{array}$ & $\begin{array}{l}1(0.8) \\
128(99.2)\end{array}$ & \\
\hline $\begin{array}{l}\text { Afatinib starting dose/day: } \\
-40 \mathrm{mg} \\
-30 \mathrm{mg} \\
-20 \mathrm{mg} \\
-10 \mathrm{mg}\end{array}$ & $\begin{array}{l}108(83.7) \\
12(9.3) \\
8(6.2) \\
1(0.8)\end{array}$ & \\
\hline $\begin{array}{l}\text { Osimertinib starting dose/day } \\
-80 \mathrm{mg} \\
-60 \mathrm{mg} \\
-40 \mathrm{mg}\end{array}$ & & $\begin{array}{l}126(97.7) \\
2(1.6) \\
1(0.8)\end{array}$ \\
\hline \multicolumn{3}{|c|}{$\begin{array}{l}\text { Data represented as } n(\%) \text {, unless otherwise stated. } \\
\text { †Includes Native American or Alaska native }(n=2[1.6 \%]) \text {, Native Hawaiian or other Pacific Islander }(n=1[0.8 \%]) \text {, no data }(n=1[0.8 \%]) \text {, other }(n=7[5.4 \%]) \text {. } \\
\text { †Data were unavailable for one patient. } \\
\text { §Two (1.6\%) patients had ECOG PS } 3 \text {. } \\
\text { ECOG PS: Eastern Cooperative Oncology Group performance status. }\end{array}$} \\
\hline
\end{tabular}

\section{Results}

Patients

A total of 129 patients from US centers were included in the analysis. Patient baseline characteristics at the start of afatinib and osimertinib therapy are summarized in Table 1. Median age at the start of afatinib treatment was 60 years (range: 52-66), 64 (49.6\%) patients were male and 28 (21.7\%) patients had Eastern Cooperative Oncology Group performance status (ECOG PS) $\geq 2$. At the start of osimertinib treatment, 57 (44.2\%) patients had ECOG PS $\geq 2$. Baseline EGFR Del19 mutations $(\mathrm{n}=92 ; 71.3 \%)$ were more common than L858R mutations $(\mathrm{n}=37$; 28.7\%). The main reason for discontinuation of afatinib was disease progression (in 125 [96.9\%] patients) and four (3.1\%) patients discontinued due to adverse events (AEs). Three patients (2.3\%) had afatinib dose interruptions.

In total, $58(50.0 \%)$ patients had discontinued osimertinib at the time of data collection. This was due to progressive disease in $54(41.9 \%)$ patients, one $(0.8 \%)$ patient discontinued due to AEs and three (2.3\%) discontinued for other reasons. No dose interruptions occurred during osimertinib treatment.

\section{Time-to-treatment failure}

TTF is summarized in Table 2. Overall median TTF for sequential afatinib and osimertinib was 28.4 months (90\% CI: 27.0-34.1; Figure 1). Median time on afatinib was 11.3 months (90\% CI: 10.3-12.0) and on osimertinib was 15.0 months (90\% CI: 13.4-16.4). Median time between the end of afatinib treatment and the start of osimertinib treatment was 22 days (range: 1-284).

In subgroups according to patient baseline and disease characteristics, long median TTF was reported in patients with EGFR Del19-positive disease (30.3 months), patients with ECOG PS 0/1 (32.7 months) and patients aged $\geq 65$ years (34.1 months; Table 2). Encouraging median TTF was also reported for patients aged $<65$ years old 


\section{Table 2. Median $\mathrm{TTF}^{\dagger}$ overall and by treatment in patients from US centers.}

\begin{tabular}{|c|c|c|c|}
\hline Patient group & Overall median TTF $(90 \% \mathrm{Cl})$ & Median TTF on afatinib $(90 \% \mathrm{Cl})$ & Median TTF on osimertinib $(90 \% \mathrm{Cl})$ \\
\hline All patients $(n=129)$ & $28.4(27.0-34.1)$ & $11.3(10.3-12.0)$ & $15.0(13.4-16.4)$ \\
\hline$<65$ years $(n=91)$ & $28.4(27.0-32.7)$ & $11.1(10.2-11.9)$ & $15.9(14.3-17.1)$ \\
\hline$\geq 65$ years $(n=38)$ & $34.1(19.9-44.5)$ & $12.2(9.2-13.1)$ & $12.3(5.7-N R)$ \\
\hline Del19 only $(n=91)$ & 30.3 (28.1-NR) & $12.0(11.4-13.1)$ & $15.9(13.8-17.6)$ \\
\hline L858R only $(n=37)$ & $24.7(14.7-27.0)$ & $10.0(9.0-10.3)$ & $8.6(5.1-14.6)$ \\
\hline ECOG PS $0 / 1(n=100)$ & 32.7 (28.4-NR) & $11.8(10.4-12.2)$ & $15.9(14.6-17.6)$ \\
\hline ECOG PS $\geq 2(n=28)$ & $22.6(16.0-27.6)$ & $10.0(7.1-10.9)$ & $11.1(4.6-14.3)$ \\
\hline
\end{tabular}

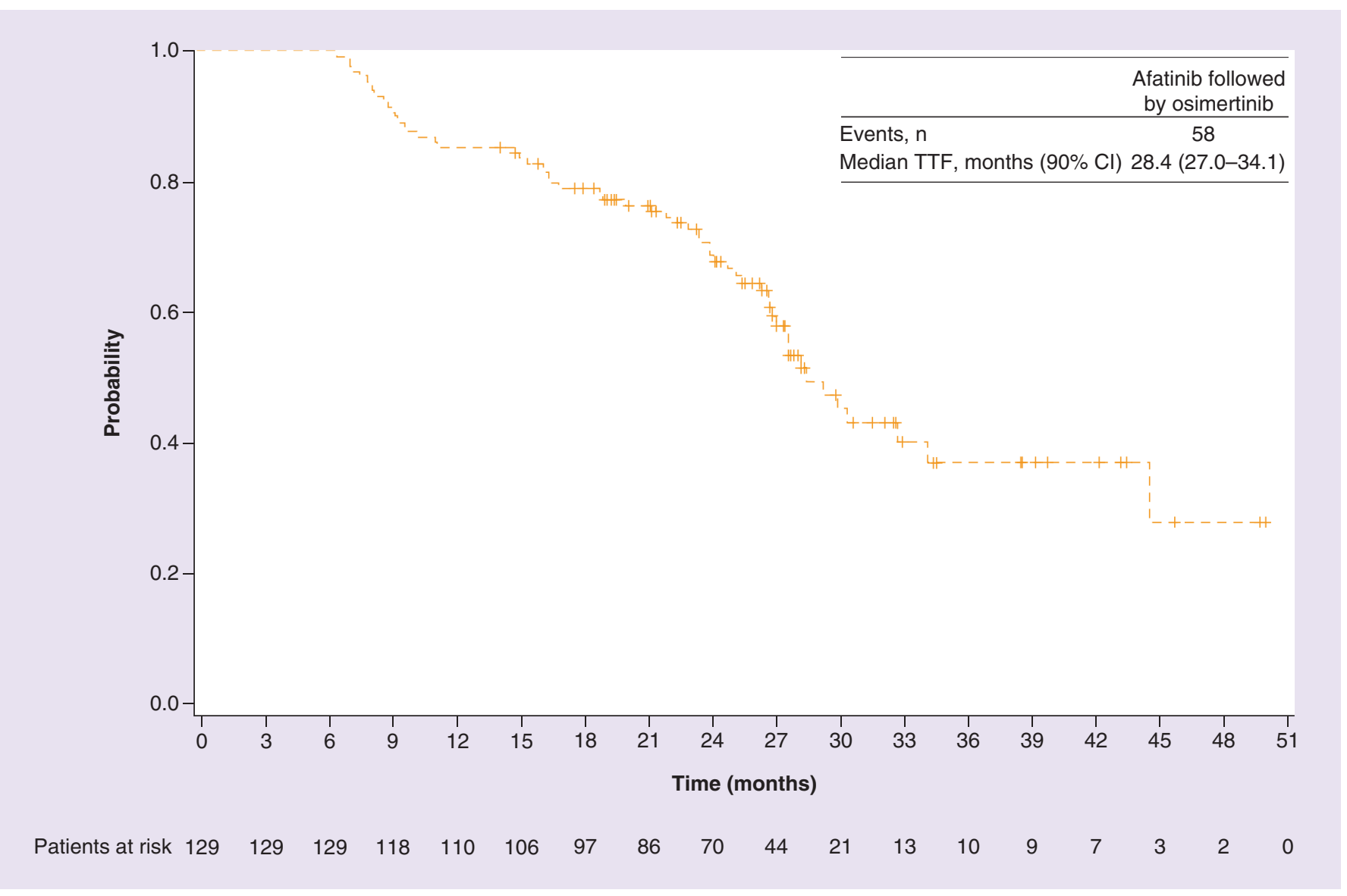

Figure 1. Overall TTF with sequential afatinib and osimertinib in US patients from the GioTag study.

TTF: Time-to-treatment failure.

(28.4 months) and patients with ECOG PS $\geq 2$ (22.6 months). Median TTF is not reported for patients with baseline brain metastases due to the small sample size $(n=7)$, but TTF for individual patients is shown in Figure 2.

Overall survival

Among all patients, the Kaplan-Meier estimate of 2-year OS was 85\% (data maturity, 35\%). Median OS was 47.6 months (90\% CI: 35.5-51.5; Figure 3).

\section{Discussion}

This subgroup analysis of the GioTag study indicates that sequential afatinib and osimertinib represents a highly effective and evidence-based treatment option for patients with EGFR mutation-positive NSCLC in the US. This 


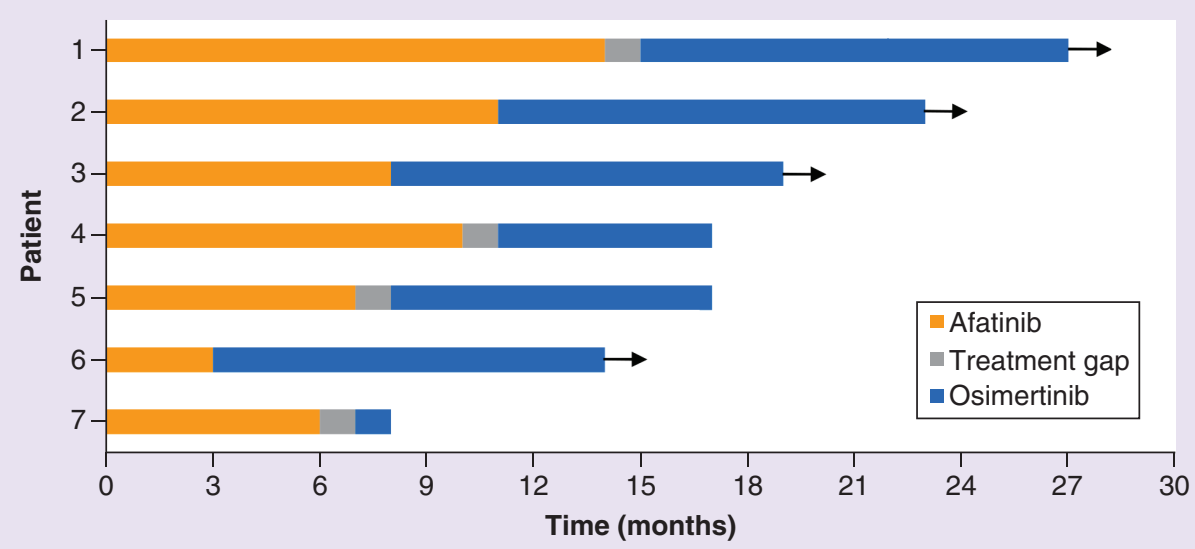

Figure 2. TTF for patients with brain metastases at baseline. Arrows denote that treatment was still ongoing at data cut-off.

TTF: Time-to-treatment failure.

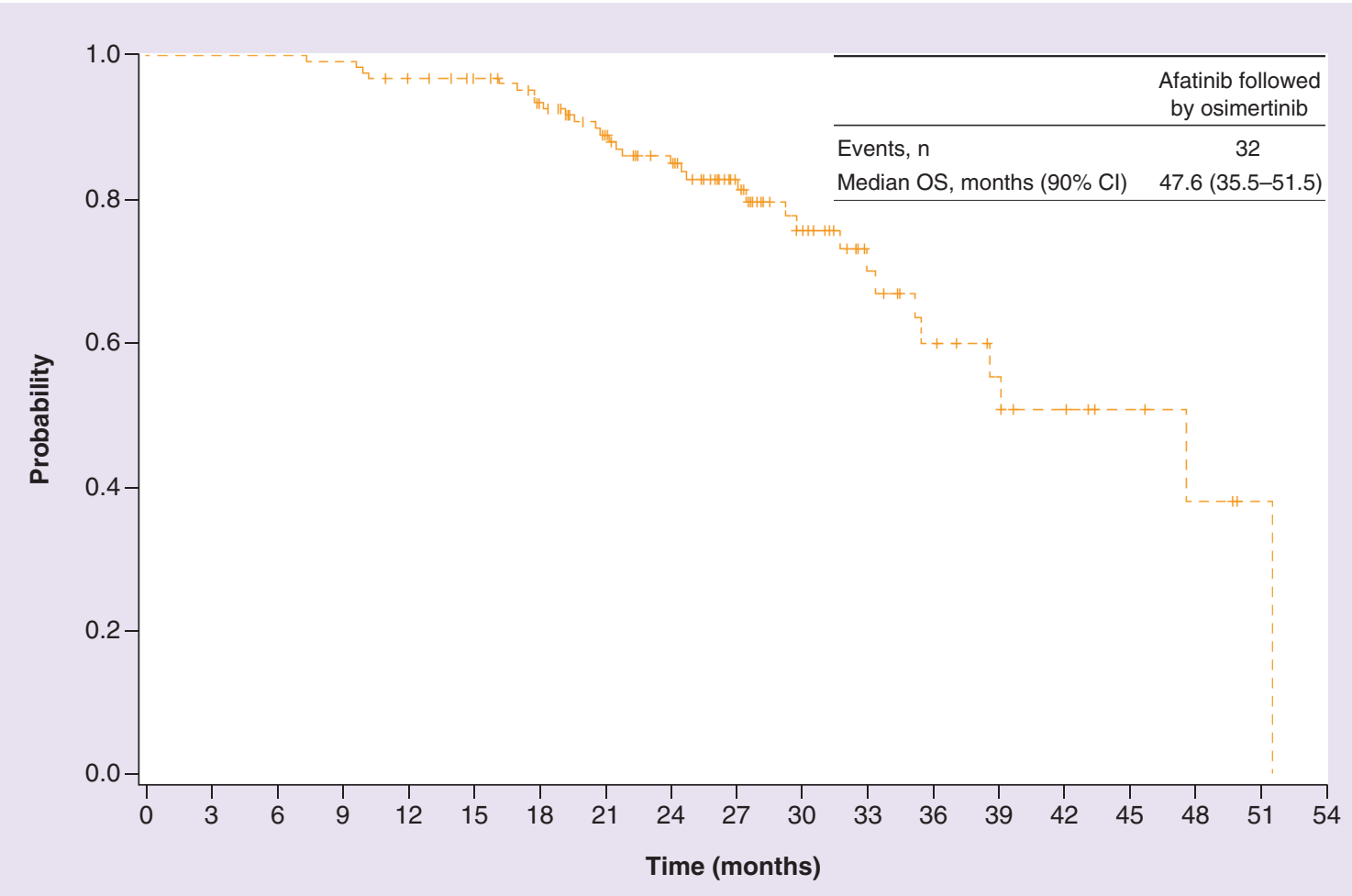

$\begin{array}{llllllllllllllllllll}\text { Patients at risk } & 129 & 129 & 129 & 128 & 123 & 118 & 109 & 94 & 81 & 57 & 36 & 23 & 17 & 12 & 9 & 5 & 3 & 1 & 0\end{array}$

Figure 3. OS with sequential afatinib and osimertinib in US patients from the GioTag study.

OS: Overall survival; US: United States.

regimen was associated with encouraging TTF in US patients overall, as well as across several subgroups, including those with EGFR Del19-positive disease and ECOG PS 0/1. Findings were generally consistent with the global primary GioTag analysis [35].

In this US subgroup analysis of GioTag, median TTF in patients with EGFR Del19 mutation-positive NSCLC was numerically longer than in all US patients (30.3 and 28.4 months, respectively). Prolonged efficacy with afatinib 
in patients with Del19-positive disease has also been reported in randomized controlled trials. In LUX-Lung 3, for example, OS was significantly improved with afatinib versus chemotherapy in patients with Del19-positive tumors (median 33.3 vs 21.1 months; $\mathrm{p}=0.0015$ ) [37]. This improvement was observed despite limited use of second-line osimertinib at the time. As the emergence of T790M is more common in EGFR Del19 (up to $75 \%$ of cases) than L858R mutation-positive disease $[25,27,28,38-40]$, sequential afatinib-osimertinib may be considered a particularly attractive treatment option for many patients with Del19-positive NSCLC.

Our findings also add to a growing body of real-world evidence demonstrating favorable outcomes with sequential afatinib-osimertinib treatment $[27,35]$. The GioTag study reported outcomes in patients who may have been excluded from randomized controlled studies, such as those with poor performance status [41]. Almost a quarter of the patients treated at US centers had ECOG PS $\geq 2$, in whom median TTF was 22.6 months, suggesting that such patients with typically poor prognosis could benefit from sequential afatinib-osimertinib. In addition, the long TTF (median 34.1 months) seen in patients aged $\geq 65$ years suggests that older age did not have a detrimental effect on treatment outcome. In this real-world study, median time on osimertinib was 15.0 months. This was longer than median PFS seen in the AURA3 clinical trial (11.0 months by independent central review) [29]. Disease progression determined by investigator assessment was the primary reason for treatment discontinuation in the GioTag study and it is possible that this long duration reflects the absence of specific disease assessment methodologies (constraints or requirements) that would normally be stipulated in clinical trial study designs. By comparison, in the clinical trial setting of AURA3, disease progression was determined by investigator assessments according to Response Evaluation Criteria in Solid Tumors, version 1.1 and also with a sensitivity analysis of PFS by blinded independent central review.

There are several limitations of the GioTag study, which have been reported in detail [35]. Two key limitations include the retrospective nature of the study and the lack of a comparator arm limiting interpretation of results. Notably, although methodological efforts were implemented to minimize the potential for selection bias (e.g., including only consecutive eligible patients from sites and limiting enrollment to a maximum of 15 patients per site), patients who died on afatinib or who were unfit or unwilling for second-line therapy were excluded. Conversely, as patients were required to have started second-line osimertinib $\geq 10$ months prior to data collection and afatinib was only approved in the US in the second half of 2013 [10], patients who derived long-term benefit from first-line afatinib may be underrepresented. In addition, data on AEs, cumulative incidence of brain metastases during treatment and patterns of disease progression (e.g., incidence of oligo-progressive disease or receipt of loco-regional treatment) were not collected for analysis. As there were only seven patients with baseline brain metastases, it was also not possible to conduct meaningful time-to-event analysis for this small subgroup.

Despite the growing body of clinical trial and real-world data on the five EGFR TKIs, the optimal sequence of TKI treatment for the individual patient warrants further investigation. There are several factors for consideration in EGFR TKI sequencing. First, data indicate that sequential afatinib-osimertinib is a feasible therapeutic strategy for many patients [34,35], especially those with EGFR Del19-positive disease, among whom techniques such as liquid biopsy ddPCR have detected high T790M emergence rates [25,27,38-40]. However, reserving osimertinib for second-line use will mean that some patients (i.e., those whose tumors accrue T790M-independent mechanisms of resistance) might never have the opportunity to benefit from the favorable efficacy and tolerability associated with osimertinib. Second, the recently reported OS benefit with osimertinib versus first-generation EGFR TKIs (median: 38.6 months [95\% CI: 34.5-41.8] vs 31.8 months [95\% CI: 26.6-36.0]; hazard ratio: 0.80 [95\% CI: 0.64-1.00]; $\mathrm{p}=0.046$ ) provides compelling evidence for the use of osimertinib in the first-line setting [16,42]. However, data from the ARCHER 1050 [15] and LUX-Lung 7 [13] clinical trials have demonstrated improved outcomes with the secondgeneration EGFR TKIs, dacomitinib and afatinib, respectively, versus the first-generation EGFR TKIs, suggesting that the benefit of osimertinib over second-generation EGFR TKIs may be less than that seen with first-generation EGFR TKIs. In addition, given the heterogeneity of resistance mechanisms to osimertinib [31] and current lack of targeted treatment options following its failure [33], sequential afatinib and osimertinib may offer a highly effective treatment option for some patients, such as those with EGFR Del19-positive disease, given the high emergence rate of T790M [25,27,38-40]. As demonstrated in the primary and US GioTag analyses [35], for some patients this sequence would yield close to 3 years of targeted therapy, delaying the need for conventional chemotherapy. A final consideration is cost-effectiveness. Although the cost-effectiveness of sequential afatinib-osimertinib has not been assessed, a recent analysis suggested that osimertinib is not a cost-effective first-line therapy for EGFR mutationpositive NSCLC when compared with first- or second-generation TKIs [43] However, it is important to note that 
further clinical trials and molecular studies are needed to resolve the outstanding questions over the optimal first-line EGFR TKI treatment strategy and identify patients for whom sequential therapy may be most appropriate.

Although the optimal first-line EGFR TKI remains under debate, investigation into novel treatment strategies is ongoing. Recent reports from Phase I trials have demonstrated preliminary antitumor activity with JNJ61186372 (a bispecific antibody to EGFR and MET) [44] and U3-1402 (an antibody-drug conjugate targeting HER3) [45] in patients whose disease has progressed after a third-generation TKI. Further data suggest consideration should be given to first-line EGFR TKI combination therapy, including antiangiogenic $[46,47]$ or chemotherapeutic agents [48-50] as the combination partners. The Phase III RELAY trial investigating erlotinib plus the VEGFR2 antagonist ramucirumab [46] and the Phase III NEJ026 study investigating erlotinib plus the anti-VEGF monoclonal antibody bevacizumab [47] have demonstrated encouraging efficacy improvements compared with EGFR TKI monotherapy treatment. More data are awaited from these and other ongoing EGFR TKI combination studies [48-50], including the AfaBev-CS study investigating afatinib in combination with bevacizumab [51].

\section{Conclusion}

Analysis of patient data from the GioTag study demonstrated a median TTF of 28.4 months in US patients treated with sequential afatinib-osimertinib in real-world clinical practice. Furthermore, encouraging TTF was reported across a broad range of subgroups, including those with Del19-positive disease and ECOG PS 0/1.

Our results demonstrate the feasibility of sequential afatinib and osimertinib for patients in the US with $E G F R$ mutation-positive NSCLC not initially presenting with de novo brain metastases or EGFR T790M-mutated disease.

\section{Summary points}

- For patients with EGFR mutation-positive non-small-cell lung cancer, second-generation (afatinib and dacomitinib) and third-generation (osimertinib) EGFR tyrosine kinase inhibitors (TKIs) have been shown to be superior to first-generation EGFR TKIs in the first-line setting.

- Resistance is inevitable to all of the EGFR TKIs; however, there is a lack of validated, targeted therapeutic options following first-line osimertinib.

- The global, observational GioTag study assessed first-line afatinib followed by osimertinib in patients who developed the T790M mutation following afatinib therapy; this analysis looks at outcomes in the subgroup of patients from the US.

- Overall, in 129 patients treated at US centers, the median time-to-treatment failure was 28.4 months $(90 \% \mathrm{Cl}$ : 27.0-34.1) and encouraging time-to-treatment failure was seen across patient subgroups, particularly those with Del19-positive disease ( $n=91$; median: 30.3 months) and Eastern Cooperative Oncology Group performance status $0 / 1$ ( $n=100$; median: 32.7 months).

- In these real-world patients in US clinical practice, median overall survival was $\mathbf{4 7 . 6}$ months.

- Consistent with the overall study population, sequential afatinib-osimertinib therapy is an effective, evidence-based treatment strategy in US clinical practice.

\section{Supplementary data}

An infographic accompanies this article and is included the end of the references section in the PDF version. To view or download this infographic in your browser, please click here: www.futuremedicine.com/doi/suppl/10.2217/fon-2020-0188

\section{Acknowledgments}

The authors are grateful to the patients, their families, the investigators and staff who participated in the study. The authors meet criteria for authorship as recommended by the International Committee of Medical Journal Editors (ICMJE). The authors received no direct compensation related to the development of the manuscript. Boehringer Ingelheim Pharmaceuticals, Inc. (BIPI) was given the opportunity to review the manuscript for medical and scientific accuracy, as well as intellectual property considerations. There is no applicable grant number.

\section{Financial \& competing interests disclosure}

The study was supported by Boehringer Ingelheim. B Feinberg is an employee of Cardinal Health. B Halmos reports grant/research support from Boehringer Ingelheim, Merck Sharp \& Dohme, AstraZeneca, Novartis, Pfizer, Bristol-Myers Squibb, Mirati, Takeda, AbbVie, Eli-Lilly and Guardant Health and consultancy roles for Boehringer Ingelheim, AstraZeneca, Genentech, Novartis, Pfizer, Bristol-Myers Squibb, Merck Sharp \& Dohme, Spectrum and Guardant Health. R Gucalp reports no potential conflicts. W Tang is an 
employee of Boehringer Ingelheim. B Moehring is an employee of Boehringer Ingelheim. MJ Hochmair reports consultancy roles for AstraZeneca, Bristol-Myers Squibb, Boehringer Ingelheim, Merck Sharp \& Dohme, Novartis, Roche and Takeda and participation in speakers' bureaus for AstraZeneca, Bristol-Myers Squibb, Boehringer Ingelheim, Merck Sharp \& Dohme, Pfizer and Roche. The authors have no other relevant affiliations or financial involvement with any organization or entity with a financial interest in or financial conflict with the subject matter or materials discussed in the manuscript apart from those disclosed.

Writing, editorial support and formatting assistance was provided by Laura Winton of GeoMed, an Ashfield company, part of UDG Healthcare plc, which was contracted and funded by Boehringer Ingelheim Pharmaceuticals, Inc. (BIPI).

Data sharing statement

The datasets generated and analyzed during the study are available from B Feinberg on reasonable request.

Open access

This work is licensed under the Attribution-NonCommercial-NoDerivatives 4.0 Unported License. To view a copy of this license, visit http://creativecommons.org/licenses/by-nc-nd/4.0/

\section{References}

Papers of special note have been highlighted as: $\bullet$ of interest; $\bullet \bullet$ of considerable interest

1. Maemondo M, Inoue A, Kobayashi K et al. Gefitinib or chemotherapy for non-small-cell lung cancer with mutated EGFR. N. Engl. J. Med. 362(25), 2380-2388 (2010).

2. Mitsudomi T, Morita S, Yatabe $\mathrm{Y}$ et al. Gefitinib versus cisplatin plus docetaxel in patients with non-small-cell lung cancer harbouring mutations of the epidermal growth factor receptor (WJTOG3405): an open-label, randomised Phase III trial. Lancet Oncol. 11(2), $121-128$ (2010).

3. Rosell R, Carcereny E, Gervais R et al. Erlotinib versus standard chemotherapy as first-line treatment for European patients with advanced EGFR mutation-positive non-small-cell lung cancer (EURTAC): a multicentre, open-label, randomised Phase III trial. Lancet Oncol. 13(3), 239-246 (2012).

4. Sequist LV, Yang JC, Yamamoto N et al. Phase III study of afatinib or cisplatin plus pemetrexed in patients with metastatic lung adenocarcinoma with EGFR mutations. J. Clin. Oncol. 31(27), 3327-3334 (2013).

5. Wu YL, Zhou C, Hu CP et al. Afatinib versus cisplatin plus gemcitabine for first-line treatment of Asian patients with advanced non-small-cell lung cancer harbouring EGFR mutations (LUX-Lung 6): an open-label, randomised Phase III trial. Lancet Oncol. 15(2), 213-222 (2014).

6. Zhou C, Wu YL, Chen G et al. Erlotinib versus chemotherapy as first-line treatment for patients with advanced EGFR mutation-positive non-small-cell lung cancer (OPTIMAL, CTONG-0802): a multicentre, open-label, randomised, Phase III study. Lancet Oncol. 12(8), 735-742 (2011).

7. US Food and Drug Administration. Tarceva. Highlights of prescribing information (2016). www.accessdata.fda.gov/drugsatfda_docs/label/2016/021743s025lbl.pdf

8. US Food and Drug Administration. Iressa. Highlights of prescribing information (2018). www.accessdata.fda.gov/drugsatfda_docs/label/2018/206995s003lbl.pdf

9. US Food and Drug Administration. Vizimpro. Highlights of prescribing information (2018). www.accessdata.fda.gov/drugsatfda_docs/label/2018/208065s011lbl.pdf

10. US Food and Drug Administration. Gilotrif. Highlights of prescribing information (2019). www.accessdata.fda.gov/drugsatfda_docs/label/2019/201292s015lbl.pdf

11. US Food and Drug Administration. Tagrisso. Highlights of prescribing information (2020). www.accessdata.fda.gov/drugsatfda_docs/label/2020/208065s016lbl.pdf

12. Park K, Tan EH, O’Byrne K et al. Afatinib versus gefitinib as first-line treatment of patients with EGFR mutation-positive non-small-cell lung cancer (LUX-Lung 7): a phase 2B, open-label, randomised controlled trial. Lancet Oncol. 17(5), 577-589 (2016).

-. Randomized Phase IIb trial demonstrating improved progression-free survival (PFS) and time-to-treatment failure with afatinib versus gefitinib in patients with epidermal growth factor receptor $(E G F R)$ mutation-positive non-small-cell lung cancer (NSCLC).

13. Paz-Ares L, Tan EH, O'Byrne K et al. Afatinib versus gefitinib in patients with EGFR mutation-positive advanced non-small-cell lung cancer: overall survival data from the Phase IIb LUX-Lung 7 trial. Ann. Oncol. 28(2), 270-277 (2017).

14. Wu YL, Cheng Y, Zhou X et al. Dacomitinib versus gefitinib as first-line treatment for patients with EGFR-mutation-positive non-small-cell lung cancer (ARCHER 1050): a randomised, open-label, phase 3 trial. Lancet Oncol. 18(11), 1454-1466 (2017).

-• Randomized Phase III trial demonstrating improved PFS with dacomitinib versus gefitinib in patients with EGFR mutation-positive NSCLC.

15. Mok TS, Cheng Y, Zhou X et al. Improvement in overall survival in a randomized study that compared dacomitinib with gefitinib in patients with advanced non-small-cell lung cancer and EGFR-activating mutations. J. Clin. Oncol. 36(22), 2244-2250 (2018). 
-• Randomized Phase III trial demonstrating improved overall survival with dacomitinib versus gefitinib in patients with $E G F R$ mutation-positive NSCLC.

16. Soria JC, Ohe Y, Vansteenkiste J et al. Osimertinib in untreated EGFR-mutated advanced non-small-cell lung cancer. N. Engl. J. Med. 378(2), 113-125 (2018).

-• Randomized Phase III trial demonstrating improved PFS with osimertinib versus gefitinib or erlotinib in patients with EGFR mutation-positive NSCLC.

17. Goss G, Tsai CM, Shepherd F et al. Osimertinib for pretreated EGFR Thr790Met-positive advanced non-small-cell lung cancer (AURA2): a multicentre, open-label, single-arm, phase 2 study. Lancet Oncol. 17(12), 1643-1652 (2016).

18. Reungwetwattana $\mathrm{T}$, Nakagawa $\mathrm{K}$, Cho BC et al. CNS response to osimertinib versus standard epidermal growth factor receptor tyrosine kinase inhibitors in patients with untreated EGFR-mutated advanced non-small-cell lung cancer. J. Thorac. Oncol. 36(33), 3290-3297 (2018).

19. Colclough N, Ballard PG, Barton P et al. Preclinical comparison of the blood brain barrier (BBB) permeability of osimertinib (AZD9291) with other irreversible next generation EGFR TKIs. Eur. J. Cancer 69, S28 (2016).

20. Ballard P, Yates JW, Yang Z et al. Preclinical comparison of osimertinib with other EGFR-TKIs in EGFR-mutant NSCLC brain metastases models and early evidence of clinical brain metastases activity. Clin. Cancer Res. 22(20), 5130-5140 (2016).

21. Yu HA, Arcila ME, Hellmann MD et al. Poor response to erlotinib in patients with tumors containing baseline EGFR T790M mutations found by routine clinical molecular testing. Ann. Oncol. 25(2), 423-428 (2014).

22. Wu JY, Yu CJ, Chang YC et al. Effectiveness of tyrosine kinase inhibitors on "uncommon" epidermal growth factor receptor mutations of unknown clinical significance in non-small cell lung cancer. Clin. Cancer Res. 17(11), 3812-3821 (2011).

23. Inukai M, Toyooka $S$, Ito $S$ et al. Presence of epidermal growth factor receptor gene T790M mutation as a minor clone in non-small cell lung cancer. Cancer Res. 66(16), 7854-7858 (2006).

24. Schuette W. Treatment of brain metastases from lung cancer: chemotherapy. Lung Cancer 45(Suppl. 2), S253-S257 (2004).

25. Yang JC, Ahn MJ, Kim DW et al. Osimertinib in pretreated T790M-positive advanced non-small-cell lung cancer: AURA study Phase II extension component. J. Clin. Oncol. 35(12), 1288-1296 (2017).

-. Phase II trial demonstrating the efficacy of osimertinib as second-line therapy.

26. Lee CK, Kim S, Lee JS et al. Next-generation sequencing reveals novel resistance mechanisms and molecular heterogeneity in EGFR-mutant non-small cell lung cancer with acquired resistance to EGFR-TKIs. Lung Cancer 113, 106-114 (2017).

27. Hochmair MJ, Buder A, Schwab S et al. Liquid-biopsy-based identification of EGFR T790M mutation-mediated resistance to afatinib treatment in patients with advanced EGFR mutation-positive NSCLC and subsequent response to osimertinib. Target. Oncol. 14(1), 75-83 (2019).

- Retrospective analysis demonstrating that up to $75 \%$ of patients with EGFR mutation-positive NSCLC may acquire the T790M mutation after first-line afatinib therapy.

28. Jenkins S, Yang JC-H, Janne PA et al. EGFR mutation analysis for prospective patient selection in two Phase II registration studies of osimertinib. J. Thorac. Oncol. 12(8), 1247-1256 (2017).

29. Mok TS, Wu YL, Ahn MJ et al. Osimertinib or platinum-pemetrexed in EGFR T790M-positive lung cancer. N. Engl. J. Med. 376(7), 629-640 (2017).

- Randomized Phase III trial demonstrating the efficacy of osimertinib as first-line therapy for patients with EGFR mutation-positive NSCLC.

30. Oxnard GR, Hu Y, Mileham KF et al. Assessment of resistance mechanisms and clinical implications in patients with EGFR T790M-positive lung cancer and acquired resistance to osimertinib. JAMA Oncol. 4(11), 1527-1534 (2018).

31. Ramalingam SS, Yang JC, Lee CK et al. Osimertinib as first-line treatment of EGFR mutation-positive advanced non-small-cell lung cancer. J. Clin. Oncol. 36(9), 841-849 (2018).

32. Ramalingam SS, Cheng Y, Zhou C et al. Mechanisms of acquired resistance to first-line osimertinib: preliminary data from the Phase III FLAURA study. Ann. Oncol. 29(Suppl. 8), Abstract LBA750 (2018).

33. Planchard D, Boyer MJ, Lee JS et al. Postprogression outcomes for osimertinib versus standard-of-care EGFR-TKI in patients with previously untreated EGFR-mutated advanced non-small cell lung cancer. Clin Cancer Res. 25(7), 2058-2063 (2019).

34. Park K, Bennouna J, Boyer M et al. Sequencing of therapy following first-line afatinib in patients with EGFR mutation-positive non-small cell lung cancer. Lung Cancer 132(2019), 126-131 (2019).

35. Hochmair MJ, Morabito A, Hao D et al. Sequential treatment with afatinib and osimertinib in patients with EGFR mutation-positive non-small-cell lung cancer: an observational study. Future Oncol. 14(27), 2861-2874 (2018).

- Primary analysis of the international, observational GioTag study of sequential afatinib-osimertinib.

36. Hochmair MJ, Morabito A, Hao D et al. Sequential afatinib and osimertinib in patients with EGFR mutation-positive non-small-cell lung cancer: updated analysis of the observational GioTag study. Future Oncol. 15(25), 2905-2914 (2019). 
37. Yang JC, Wu YL, Schuler M et al. Afatinib versus cisplatin-based chemotherapy for EGFR mutation-positive lung adenocarcinoma (LUX-Lung 3 and LUX-Lung 6): analysis of overall survival data from two randomised, phase 3 trials. Lancet Oncol. 16(2), 141-151 (2015).

38. Wu SG, Liu YN, Tsai MF et al. The mechanism of acquired resistance to irreversible EGFR tyrosine kinase inhibitor-afatinib in lung adenocarcinoma patients. Oncotarget 7(11), 12404-12413 (2016).

39. Ke EE, Zhou Q, Zhang QY et al. A higher proportion of the EGFR T790M mutation may contribute to the better survival of patients with exon 19 deletions compared with those with L858R. J. Thorac. Oncol. 12(9), 1368-1375 (2017).

40. Matsuo N, Azuma K, Sakai K et al. Association of EGFR exon 19 deletion and EGFR-TKI treatment duration with frequency of T790M mutation in EGFR-mutant lung cancer patients. Sci. Rep. 6, 36458 (2016).

41. Park K, Wan-Teck Lim D, Okamoto I et al. First-line afatinib for the treatment of EGFR mutation-positive non-small-cell lung cancer in the 'real-world' clinical setting. Ther. Adv. Med. Oncol. 11, 1758835919836374 (2019).

42. Ramalingam SS, Vansteenkiste J, Planchard D et al. Overall survival with osimertinib in untreated, EGFR-mutated advanced NSCLC. N. Engl. J. Med. 382(1), 41-50 (2020).

- Randomized Phase III trial demonstrating superior overall survival with first-line osimertinib versus erlotinib/gefitinib.

43. Aguiar PN Jr, Haaland B, Park W et al. Cost-effectiveness of osimertinib in the first-line treatment of patients with EGFR-mutated advanced non-small cell lung cancer. JAMA Oncol. 4(8), 1080-1084 (2018).

44. Haura E, Cho B, Lee J et al. JNJ-61186372 (JNJ-372), an EGFR-cMet bispecific antibody, in EGFR-driven advanced non-small cell lung cancer (NSCLC). J. Clin. Oncol. 37(Suppl. 15), Abstract 9009 (2019).

45. Janne $\mathrm{P}$, Yu H, Johnson M et al. Safety and preliminary antitumor activity of U3-1402: a HER3-targeted antibody drug conjugate in EGFR TKI-resistant, EGFRm NSCLC. J. Clin. Oncol. 37(Suppl. 15), Abstract 9010 (2019).

46. Nakagawa K, Garon EB, Seto T et al. RELAY: a multinational, double-blind, randomized phase 3 study of erlotinib (ERL) in combination with ramucirumab (RAM) or placebo (PL) in previously untreated patients with epidermal growth factor receptor mutation-positive (EGFRm) metastatic non-small cell lung cancer (NSCLC). J. Clin. Oncol. 37(Suppl. 15), Abstract 9000 (2019).

47. Saito H, Fukuhara T, Furuya N et al. Erlotinib plus bevacizumab versus erlotinib alone in patients with EGFR-positive advanced non-squamous non-small-cell lung cancer (NEJ026): interim analysis of an open-label, randomised, multicentre, phase 3 trial. Lancet Oncol. 20(5), 625-635 (2019).

48. Wen M, Xia J, Sun Y et al. Combination of EGFR-TKIs with chemotherapy versus chemotherapy or EGFR-TKIs alone in advanced NSCLC patients with EGFR mutation. Biologics 12, 183-190 (2018).

49. Noronha V, Joshi A, Patil VM et al. Phase III randomized trial comparing gefitinib to gefitinib with pemetrexed-carboplatin chemotherapy in patients with advanced untreated EGFR mutant non-small cell lung cancer (gef vs gef+C). J. Clin. Oncol. 37(Suppl. 15), 9001 (2019).

50. Nakamura A, Inoue A, Morita $S$ et al. Phase III study comparing gefitinib monotherapy (G) to combination therapy with gefitinib, carboplatin and pemetrexed (GCP) for untreated patients (pts) with advanced non-small cell lung cancer (NSCLC) with EGFR mutations (NEJ009). J. Clin. Oncol. 36(Suppl. 15), 9005 (2018).

51. Ninomiya $\mathrm{T}$, Ishikawa $\mathrm{N}$, Inoue $\mathrm{K}$ et al. Phase 2 study of afatinib alone or combined with bevacizumab in chemonaive patients with advanced non-small-cell lung cancer harboring EGFR mutations: afaBev-CS study protocol. Clin. Lung Cancer 20(2), 134-138 (2019). 
Original short communication article: Making the case for EGFR TKI sequencing in EGFR mutation-positive NSCLC: a GioTag study US patient analysis. Feinberg, et al. Future Oncology 2020

\section{THE GIOTAG STUDY: FOCUS ON PATIENTS IN THE USA}

\section{Afatinib followed by osimertinib for EGFRm + NSCLC}

WHAT IS THE

GIOTAG STUDY?

EGFRm + = with an EGFR mutation NSCLC $=$ non-small-cell lung cancer 1. Hochmair MJ, et al. Future Oncol. 2018; 14; 2861-74

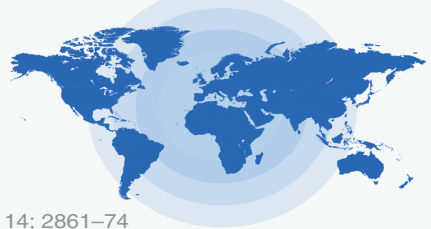

(6) A global study 1

(D) Looking at medical records of patients with EGFRm+ advanced NSCLC

$\rightarrow$ Specifically patients who received afatinib followed by osimertinib

\section{WHY AFATINIB FOLLOWED BY OSIMERTINIB?}
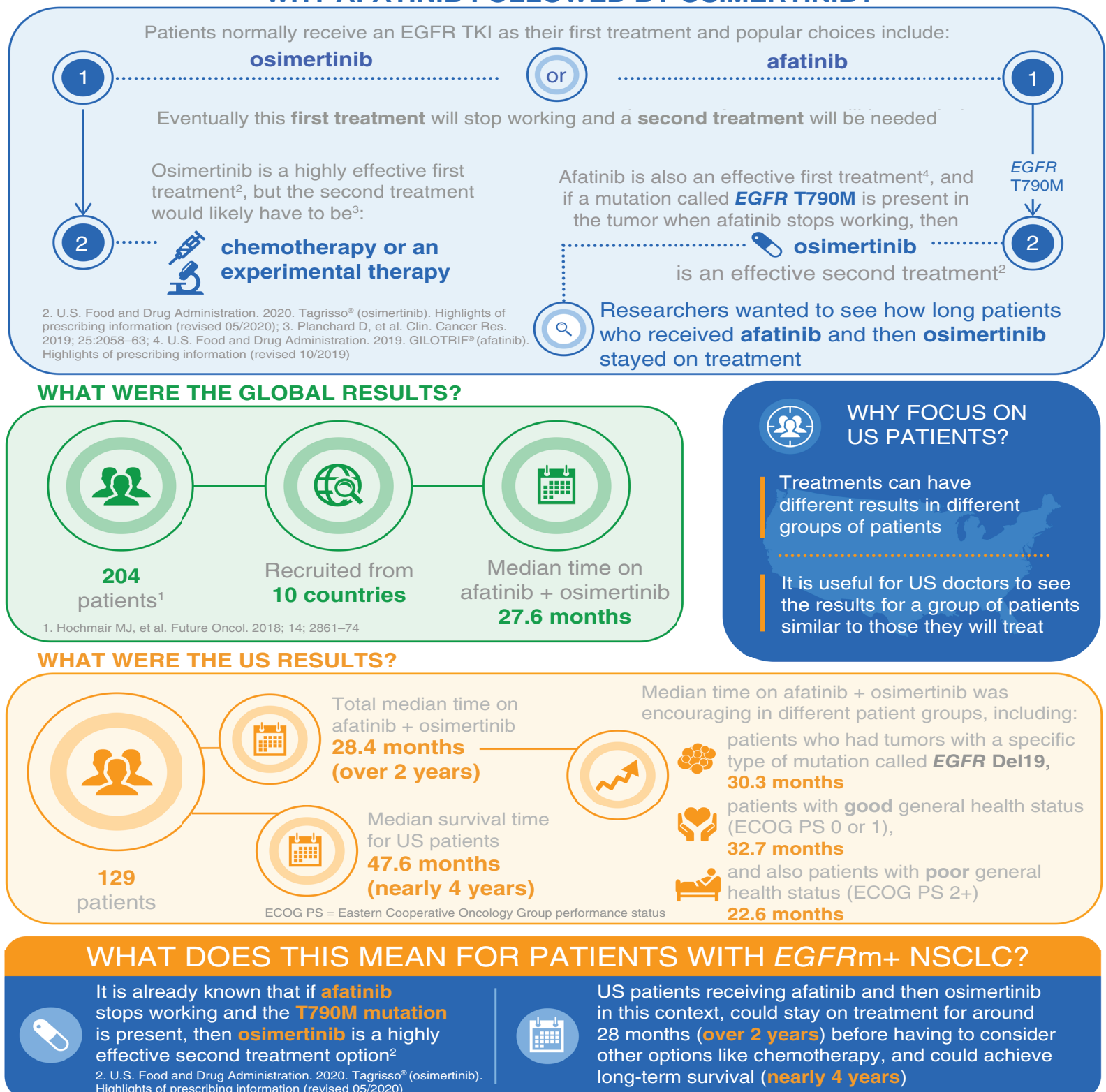
22.6 months

Treatments can have different results in different groups of patients

It is useful for US doctors to see the results for a group of patients similar to those they will treat

The authors meet criteria for authorship as recommended by the International Committee of Medical Journal Editors (ICMJE). The authors received no direct compensation related to the development of the materials. Writing, editorial support, and formatting assistance for this material was provided by Laura Winton, of GeoMed, an Ashfield company, part of UDG Healthcare plc, which was contracted and compensated by Boehringer Ingelheim Pharmaceuticals Inc. (BIPI), for these services. BIPI was given the opportunity to review the content for medical and scientific accuracy as well as intellectual property considerations 